\title{
An orientation-contingent achromatic aftereffect
}

\author{
LORRAINE G. ALLAN and NADIA TIRIMACCO \\ McMaster University, Hamilton, Ontario, Canada
}

\begin{abstract}
During a 20-min adaptation period, a pattern of black horizontal bars on a light gray background was alternated with a pattern of black vertical bars on a dark gray background. The appearance of the homogeneous gray background of the test figure was contingent on bar orientation: the background of the horizontal bars was reported as darker than the background of the vertical bars.
\end{abstract}

Orientation-contingent color aftereffects, originally reported by McCollough (1965), are readily demonstrated. During an adaptation period of several minutes, an observer inspects two patterns that alternate every few seconds. For example, one pattern could be black horizontal bars on a green background and the other could be black vertical bars on a magenta background. Following such adaptation, complementary color aftereffects contingent on bar orientation are noted. In the above example, the achromatic background of horizontal bars would appear pinkish and the achromatic background of vertical bars would appear greenish.

Tirimacco (1985) reported that subjects who were categorized as color deficient on the basis of the Ishihara (1982) test did not differ significantly from normal controls with regard to the size of the McCollough effect. She noted a possible confounding factor in her study, namely that the magenta and the green were of different luminances, and suggested that her result might be, at least in part, attributable to an orientation-contingent achromatic aftereffect. We report an experiment that investigated this possibility. We alternated, during adaptation, a pattern of black horizontal bars on a light gray background with a pattern of black vertical bars on a dark gray background. We were interested in the appearance of a test figure composed of horizontal and vertical bars on a homogeneous gray background.

\section{METHOD}

\section{Subjects}

The subjects were 4 male and 6 female students with no previous experience in contingent aftereffect tasks. They were enrolled in an introductory psychology course at McMaster University and received course credit for their participation.

\section{Apparatus}

The adaptation and test stimuli were presented on an Electrohome RGB color monitor (Model ECM 1301) driven by a Vectrix color graphics

This research was supported by a grant from the Natural Sciences and Engineering Research Council of Canada to the first author, and constitutes part of an honors Bachelor of Science thesis by the second author. Requests for reprints should be sent to Lorraine G. Allan, Department of Psychology, McMaster University, Hamilton, Ontario, Canada L8S 4K1. computer (Model VX384). The monitor was located $120 \mathrm{~cm}$ from the subject and the stimuli were $11 \mathrm{~cm}$ square, subtending $5.27^{\circ}$ of visual angle. Temporal parameters, stimulus presentation, and recording of responses were controlled by a Commodore 64 computer.

The color on the Electrohome RGB monitor is the result of a combination of red, green, and blue $(R, G, B)$. When each input is at its maximum value $(R=G=B=255)$, the result is white. Decreasing all three values by equal amounts produces various shades of gray, from light to dark. When each input is at its minimum value $(R=G=B=0)$, the result is black. Changing the relative proportions of $R, G$, and $B$ produces various hues at different saturations.

\section{Stimuli}

The adaptation patterns were composed of 33 bars, either horizontal or vertical. Sixteen of the bars were black $(R=G=B=0)$, each bar subtending $.167^{\circ}$ of visual angle. The horizontal black bars alternated with light gray $(R=G=B=200)$ bars and the vertical black bars alternated with dark gray $(R=G=B=80)$ bars, each gray bar subtending $.153^{\circ}$ of visual angle.

The test pattern was an $11-\mathrm{cm}$ square divided into quadrants. Horizontal and vertical bars, of the same spatial frequency as in the adaptation patterns, appeared in diagonally opposite quadrants. The amount of $R, G$, and $B$ provided to each diagonally opposite pair of quadrants could be adjusted by the operation of a joystick connected to the Commodore 64 computer.

\section{Aftereffect Measurement}

The magnitude of the orientation-contingent achromatic aftereffect was determined with a computer colorimeter. At the outset of a determination, two diagonally opposite quadrants were clearly dark gray $(R=G=B=108)$, and the other two were clearly light gray $(R=G=B=172)$. The subject was instructed to adjust the display so that the entire figure appeared to be one shade of gray. The computer was programmed so that movement of the joystick in one direction lightened the grayness of one pair of diagonally opposite quadrants in 2-unit steps and simultaneously darkened the grayness in the other pair of quadrants in 2-unit steps. Movement of the joystick in the other direction had the opposite effect.

In the absence of an achromatic color aftereffect, a subject should adjust the pattern until, in all four quadrants, $R=G=B=140$, the value midway between the two adaptation values. This setting, however, would appear as a mismatch if an achromatic aftereffect were present. Specifically, for a homogeneous gray test figure to be seen following adaptation with the dark gray verticals and light gray horizontals used in this experiment, the setting would be greater than 140 for the horizontal quadrants and would be less than 140 for the vertical quadrants.

\section{Procedure}

Subjects were tested individually in a darkened room, with the experimenter present throughout the session. There were four phases: baseline measurement, adaptation, test, and verbal report. 
Baseline measurement. Prior to adaptation, subjects were presented with the test pattern on six occasions. On three of the baseline determinations, the upper left and lower right quadrants were initially dark gray, and the other diagonally opposite quadrants were light gray. This was reversed on the other three baseline determinations. For each determination, the subject was instructed to manipulate the joystick until the pattern appeared to be a homogeneous gray. The mean of the last four settings provided the baseline measure.

Adaptation. Following baseline determination, subjects were adapted for $20 \mathrm{~min}$, with the two adaptation patterns, horizontal black bars on a light gray background and vertical black bars on a dark gray background, alternating every $5 \mathrm{sec}$. Adaptation was followed by $2 \mathrm{~min}$ in normal room illumination prior to the test phase.

Test. The test phase was identical to the baseline phase except that there were only four determinations. The difference between the mean baseline measure and the mean test measure constituted the magnitude of the achromatic aftereffect. Consider the following example of a calculation of the magnitude for a given subject. During baseline determination, the mean setting of the colorimeter would be near $R=G=B=140$, say $R=G=B=138$ for the vertical quadrants and hence $R=G=B=142$ for the horizontal quadrants. If an aftereffect were induced during adaptation, there would be a decrease in the setting for the verticals, for example, a mean setting of $R=G=B=134$, and a corresponding increase to $\mathrm{R}=\mathrm{G}=\mathrm{B}=146$ in the mean setting for the horizontals. If we let $H V_{B}$ represent the difference between the horizontal setting during baseline $\left(H_{B}=142\right)$ and the vertical setting during baseline $\left(V_{B}=138\right)$, then, for this example, $H V_{B}=4$. Similarly, if $H V_{T}$ represents the difference between the horizontal setting during test $\left(H_{T}=146\right)$ and the vertical setting during test $\left(V_{T}=134\right)$, then $H V_{T}=12$. The measure of the achromatic aftereffect would be $H V_{T}-H V_{B}=8$.

Verbal report. A test pattern, in which $R=G=B=140$ in all four quadrants, was presented. The subject was asked whether the pattern was a homogeneous gray, and, if the response was "no," was asked to describe the pattern. The subject was also asked to rotate his/her head through $90^{\circ}$ and to report any changes.

\section{RESULTS AND DISCUSSION}

If an achromatic aftereffect were established during adaptation, the background of the horizontal bars during test would appear darker than the background of the vertical bars. Therefore, in order for the pattern to appear homogeneous, the test setting for the horizontal quadrants would be a higher value than the baseline setting $\left(H_{T}>H_{B}\right)$ and the test setting for the vertical quadrants would be a lower value than the baseline setting $\left(V_{T}<V_{B}\right)$. This was the case for each subject. The mean $H V_{T}$ value was 8.6 and the mean $H V_{B}$ value was 1.9. Thus, relative to baseline, subjects set the horizontal background a lighter gray than the vertical background.
The verbal reports were consistent with the colorimeter settings. Nine of the 10 subjects perceived an achromatic aftereffect when viewing the homogeneous gray test pattern. They reported that the vertical quadrants were lighter than the horizontal quadrants. They also reported that the grayness experienced reversed when the head was rotated $90^{\circ}$ and that the pattern did appear to be a homogeneous gray when the head was tilted $45^{\circ}$.

The results clearly indicate that an achromatic aftereffect contingent on orientation can be established. The only reference to an achromatic aftereffect of which we are aware is that by Skowbo and Clynes (1977). They suggested that presentation of achromatic grids after adaptation results in a weakened color aftereffect "because an 'achromatic McCollough effect' builds up, reducing the assessed strength of the chromatic effect" (p. 181). They noted that evidence for this aftereffect had not been documented in the literature. We have now provided this evidence, but we do not see how it accounts for the weakened color aftereffect. First, in Skowbo and Clynes's (1977) situation, as in all extinction preparations, the achromatic background of the horizontal bars and of the vertical bars was the same gray. Second, although the background in the Skowbo and Clyne's study was physically achromatic, it was not achromatic for the subject. After adaptation, the achromatic grids would be colored for the subject.

\section{REFERENCES}

IsHIHARA, S. (1982). The series of plates designed as a test for colour blindness. Japan: Kanehara Shuppan.

McCollough, C. (1965). Color adaptation of edge-detectors in the human visual system. Science, 149, 1115-1116.

Skowbo, D., \& Clynes, N. (1977). Decline and revival of McCollough effects following inspection of achromatic gratings. Perception \& Psychophysics, 21, 180-182.

TiRIMAcco, N. (1985). An achromatic aftereffect contingent on line orientation. Unpublished honors Bachelor of Science thesis, McMaster University, Hamilton, Ontario, Canada.

(Manuscript received for publication September 15, 1986.) 